\title{
Lightning Protection Performance Assessment of Transmission Line Based on ATP model Automatic Generation
}

\author{
Luo Hanwu', Li Mengke ${ }^{1}$, Xu Xinyao', Cui Shigang ${ }^{1}$, Han Yin ${ }^{1}$, Yan Kai ${ }^{1}$, Wang Jing ${ }^{1}$, Le Jian ${ }^{2 a}$ \\ ${ }^{1}$ State Grid East Inner Mongolia Electric Power Maintenance Company, Tongliao 028000, Neimenggu Province, \\ China \\ ${ }^{2}$ School of Electrical Engineering, Wuhan University, Wuhan 430072, China
}

\begin{abstract}
This paper presents a novel method to solve the initial lightning breakdown current by combing ATP and MATLAB simulation software effectively, with the aims to evaluate the lightning protection performance of transmission line. Firstly, the executable ATP simulation model is generated automatically according to the required information such as power source parameters, tower parameters, overhead line parameters, grounding resistance and lightning current parameters, etc. through an interface program coded by MATLAB. Then, the data are extracted from the generated LIS files which can be obtained by executing the ATP simulation model, the occurrence of transmission lie breakdown can be determined by the relative data in LIS file. The lightning current amplitude should be reduced when the breakdown occurs, and vice the verse. Thus the initial lightning breakdown current of a transmission line with given parameters can be determined accurately by continuously changing the lightning current amplitude, which is realized by a loop computing algorithm that is coded by MATLAB software. The method proposed in this paper can generate the ATP simulation program automatically, and facilitates the lightning protection performance assessment of transmission line.
\end{abstract}

\section{Introduction}

Transmission line plays a key role in the transmission of electrical power from power plant to endusers, its reliability has an immensely impact on the safe and reliable operation of the whole power system[1]. The transmission line corridor in China is characterized by the diversity and multiplicity in environment due to the vast land area and complex topography and geomorphology distribution[2,3]. Many transmission lines, especially those with rated voltage level is $110 \mathrm{kV}$ and above, are subjected to the test of complex terrain and climate[4]. Lightning accident is a kind of severe natural disaster, its frequent occurrence is more likely to cause the flashover accident of transmission line, thus seriously threatens the safe and reliable operation of power system[5,6]. Therefore, the lightning protection performance of transmission line and the performance evaluation is the foundation for the design of lightning protection measures[7]. In this sense, to evaluate the lightning protection level using computer and automation technologies to obtain the lightning withstand level at different places is the

\footnotetext{
${ }^{a}$ Corresponding author : lej01@ mails.tsinghua.edu.cn 
key aspect for the comprehensive assessment of the lightning protection performance of transmission line $[8,9]$.At present, the methods to evaluate the lighting performance of transmission line include regulation calculate method and simulation analysis method[10,11]. Regulation calculate method that is based on operation experience has been widely used in engineering due to fact that it is simple and easy to use, However it applies inductance model to handle the lightning current pathway on the tower and take no account on the wave process of the tower, thus lead to large error between the calculation and actual result[12]. The simulation analysis method need to establish simulation model at first, and then run the model on computer to obtain the lighting performance. The lighting back-strike model involves the choose of the method to calculate the lightning counter-attack withstand level, while the shielding failure model involves the choose of analysis model to calculate the shielding failure rate and the shielding failure trip rate[13,14]. It need to establish the simulation model for a transmission line manually, and then change the lightning current manually many times to obtain the initial breakdown current of the transmission line, which is a complicated and time consuming work.

In this paper, a method to solve the initial breakdown current of transmission line by integrating the ATP and MATLAB simulation software effectively is presented. It can improve the automatic level and accuracy in evaluating the lightning protection performance of transmission line.

The remainder of this paper is structured as follow: In Section II, the characteristics and simulation process of ATP and MATLAB is introduced briefly for reference purpose. The analysis on the structure of ATP/EMTDC simulation program and the principle of the automatic simulation program creation is given in Section III. The framework and process of the method that automatically solves the initial breakdown current of transmission line, and an example to illustrate the application and validate the effectiveness of the proposed method are present in Section IV and V, respectively. Finally in Section VI, the main findings of the paper are summarized.

\section{Simulation Software Introduction}

ATP(Alternative Transients Program) /EMTDC simulation software is a kind of electro-magnetic transient analysis program that has perfect compatibility to run in multi-types of platforms and has been widely applied in power system simulation.

ATPDraw, which is a preliminary data processing part of ATP, is used to generate the input date file for ATP/EMTDC program with correct format. Essentially, it is a kind of visualized programming interface through which many simulation models can be established intuitively and graphically. The established simulation mode in graphical form will be translated by compiler into ATP file(*.atp) that is in text form, and this ATP file is the actual simulation model that can be executed by ATP/EMTDC program.

MATLAB software, which is developed by Math Works corporation in the United States, is one of the most widely used mathematical simulation software for its powerful mathematical analysis functions. MATLAB can be used for the purpose of image fitting and rendering, numerical analysis and calculation, also as an interface to interact with many other software.

In this paper, the program used to automatically create ATP simulation model and solve the initial lightening breakdown voltage of transmission line is coded by MATLAB for its convenience and multiple interface in interacting with other program.

\section{ATP-EMTP Model Auto-creation}

\subsection{ATP file parsing}

The code in ATP-EMTP file typically is in the type of comment line and code line, and the role of the comment line is to explain the procedures and instructions, thus do not affect the function of simulation model. 
The code line is distributed in different data cards, which are connected in sequence and forms the executable ATP simulation model. According to the order of sequence in ATP file, these data cards are: starting data card, switch data card, MODEL data card, TACS data card branch data card, power data card, output data card and blank data card. Fig.1 shows the content of a typical ATP file containing above data cards.

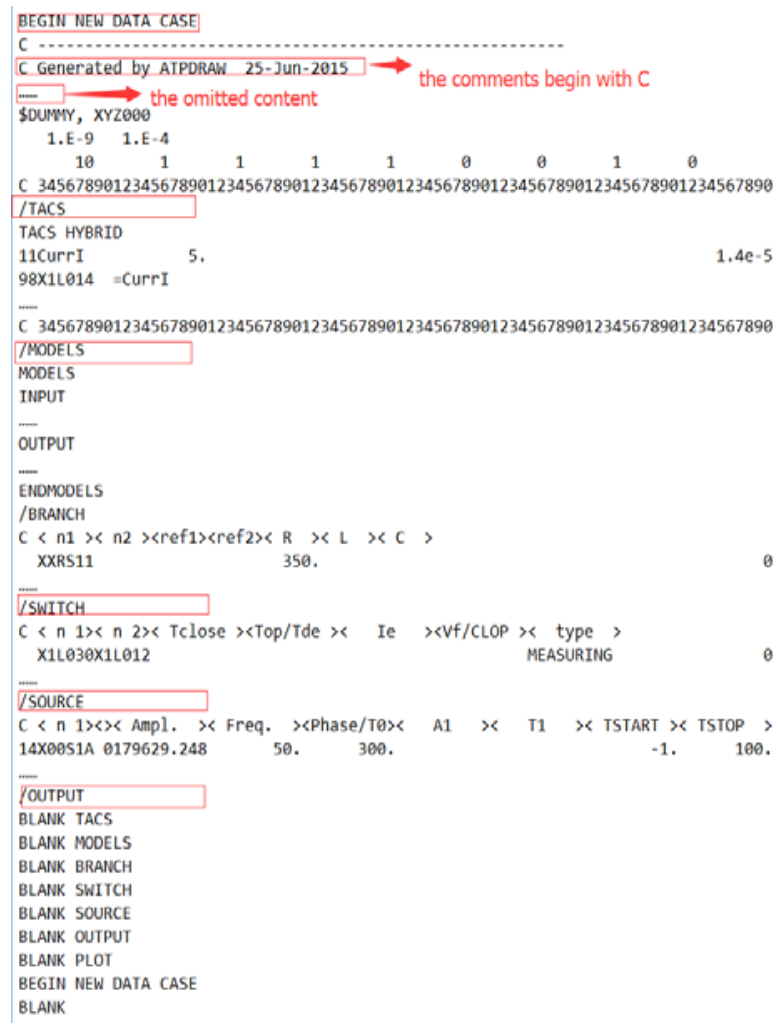

Figure 1. Content of a typical ATP file

Starting data card. This card contains the initial statement and some simulation parameters, including simulation step size, simulation time, plot step size and so on.

TACS(Transient analysis of Control Systems) data card. This cad is used in control system transient simulation.

MODEL data card. The card is a custom component that can be implemented with different functions.

Branch data card. The role of this card is to number the elements in simulation model in sequence to determine the connection relationship between them. The code to call for Lib file is at the end of this card. The Lib file is the library file of the overhead line model. The LIB file of overhead line needs to be created before the creation of ATP file.

Switch data card. The function of this card is to set the open/close status of individual switch in simulation model.

Power data card. The function of the card is to set the parameters of the power source in simulation model.

Output data card. The function of the card is to output the values of the variables measured by voltage or current meter.

Blank data card. This card is used to represent the end of the simulation model. Any complete ATP file should be ended with this card, the ATP program will terminate when it finds an ATP model without blank data card. 


\subsection{Auto-creation implementation}

By the above analysis on the structure of ATP file, the auto-creation of ATP file can be achieved by the following steps as shown in Fig.2: 1) Reading required data for the establishing of simulation model from data files, and processing these data to create *.mat file; 2) Reading date related to overhead line to create *.lib file; 3) Generating each data card used in ATP file; 4) Connecting the data cards in right sequence to create ATP file.

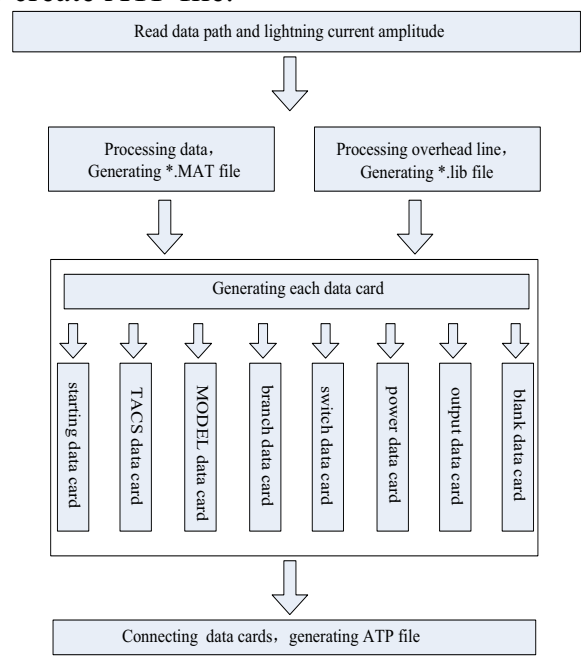

Figure 2. ATP file auto-generation flow

ATP-EMTP program has very strict requirement on the format of ATP file, for example, different types of component may has different character length, and the requirement on the arrangement of characters is very strict. The input data should be translated in to string with certain length. When the length of the input data is too long, it should be converted into the form of scientific notation and saved in the form of a string. While when the data length is not long enough, blanks should be added at the beginning of the data and then saved as a string .

Overhead line model is handled specially in ATP file. It should be proceed by LCC module provided by ATP program to create LIB file that can be called by ATP file. Fig.3 shows the processing of overhead line model.

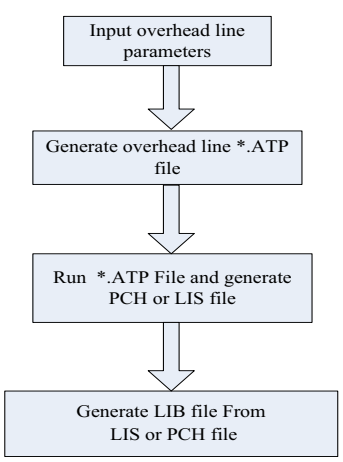

Figure 3. Generation of the overhead line LIB file

\section{Solution of the Initial Breakdown Voltage}

Once the ATP simulation model is established automatically, the initial breakdown current of transmission line can be solved by using the steps shown in Fig.4. 


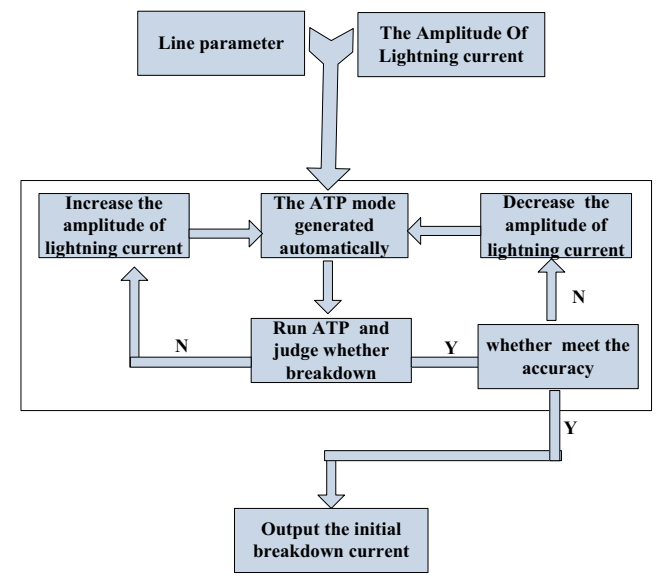

Figure 4. Process for solving initial breakdown current

The principle of this algorithm is to set a initial amplitude for lightning current, and create the ATP simulation model automatically according to the line parameters, then run the simulation model and obtain the result. If no breakdown occurs, then increase the lightning current amplitude, otherwise decrease, then create the simulation model and run again it automatically to find whether the breakdown occurs or not. Repeat above process until the amplitude difference between the two adjacent lightning current is less than the preset threshold, then the initial breakdown current amplitude is solved.

\section{Application of the Proposed Method}

Fig.5 shows the parameters of the components in simulation model of overhead line lighting strike, and Fig.6 shows the part code of the created simulation model according to these parameters. Executing the created model automatically and automatically change the lightning current amplitude every time until the initial breakdown current of transmission line is solved.

\begin{tabular}{|c|c|c|c|c|c|c|c|c|c|}
\hline \multirow{2}{*}{ SOURCE PARAMETES } & 179629.2478 & 50 & 30 & 0 & & & & & \\
\hline & 179629.2478 & 50 & 300 & 0 & & & & & \\
\hline \multirow{18}{*}{ TOWER PARAMETERS } & 1 & 113.3 & 225000000 & 0.7 & 113.3 & 225000000 & 0.7 & & \\
\hline & 1 & 334.1 & 225000000 & 4.07 & 334.1 & 225000000 & 4. 07 & & \\
\hline & 1 & 107.4 & 225000000 & 4 & 107.4 & 225000000 & 4 & & \\
\hline & 1 & 251.2 & 225000000 & 2.75 & 251.2 & 225000000 & 2.75 & & \\
\hline & 1 & 102.5 & 225000000 & 5 & 102.5 & 225000000 & 5 & & \\
\hline & 1 & 102.8 & 225000000 & 15.7 & 925.8 & 225000000 & 23.55 & & \\
\hline & 2 & 113.3 & 225000000 & 0.7 & 113.3 & 225000000 & 0.7 & & \\
\hline & 2 & 334.1 & 225000000 & 4. 07 & 334.1 & 225000000 & 4.07 & & \\
\hline & 2 & 107.4 & 225000000 & 4 & 107.4 & 225000000 & 4 & & \\
\hline & 2 & 251.2 & 225000000 & 2.75 & 251.2 & 225000000 & 2.75 & & \\
\hline & 2 & 102.5 & 225000000 & 5 & 102.5 & 225000000 & 5 & & \\
\hline & 2 & 102.8 & 225000000 & 15.7 & 925.8 & 225000000 & 23.55 & & \\
\hline & 3 & 113.3 & 225000000 & 0.7 & 113.3 & 225000000 & 0.7 & & \\
\hline & 3 & 334.1 & 225000000 & 4.07 & 334.1 & 225000000 & 4.07 & & \\
\hline & 3 & 107.4 & 225000000 & 4 & 107.4 & 225000000 & 4 & & \\
\hline & 3 & 251.2 & 225000000 & 2.75 & 251.2 & 225000000 & 2.75 & & \\
\hline & 3 & 102.5 & 225000000 & 5 & 102.5 & 225000000 & 5 & & \\
\hline & 3 & 102.8 & 225000000 & 15.7 & 925.8 & 225000000 & 23.55 & & \\
\hline \multirow{4}{*}{ 'OWER LINE PARAMETERS } & 5 & 2328.6 & 0.005 & 0.17 & 0 & 10 & 10 & 500000 & 50 \\
\hline & 5 & 2328.6 & 0.005 & 0.18 & 0 & 10 & 10 & 500000 & 50 \\
\hline & 5 & 2328.6 & 0.005 & 0.18 & 0 & 10 & 10 & 500000 & 50 \\
\hline & 5 & 2328.6 & 0.005 & 0.18 & 0 & 10 & 10 & 500000 & 50 \\
\hline LIGHTNING PARAMETERS & -50000 & 2. $6 \mathrm{E}-06$ & -1000 & 0.00005 & 0.00001 & 1 & -1 & 2 & 2 \\
\hline \multirow{3}{*}{ GROUND PARAMETERS } & 24 & 500 & 2500 & & & & & & \\
\hline & 24 & 500 & 2500 & & & & & & \\
\hline & 24 & 500 & 2500 & & & & & & \\
\hline
\end{tabular}

Figure 5. Parameters of the component in model 


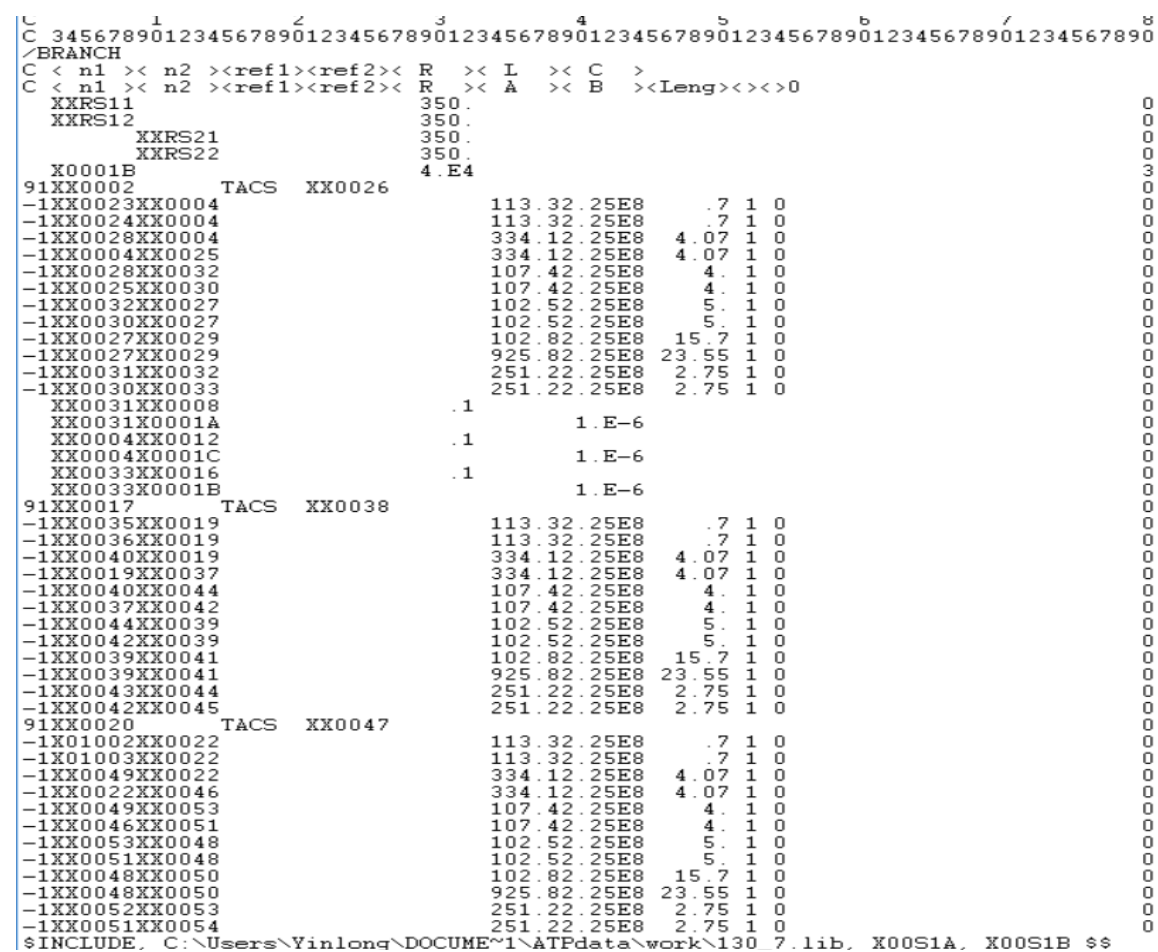

Figure 6. Part of code created automatically

\section{Conclusions}

In this paper, a method of automatic generation of ATP simulation model is put forward, which can solve the problems such as heavy workload and easy to make mistake in establishing the ATP simulation model. On this basis, the method of the iterative solution of the initial breakdown current of overhead transmission line is proposed based on the presented simulation model automatic generation method, which can improve the efficiency and accuracy. The proposed method can be applied to other occasions based on ATP simulation, which has high practical value and application prospect.

\section{References}

1. Haiyang Wang. Journal of jinling institute of technology. 27(4), 17-22,(2011),:

2. Deji Shang. The public science and technology.5,120-122,(2010).

3. Gang Chen, Zhang Peichai.. Relay. 34(22),5-8,( 2006).

4. Yan Wang, Guo Huaide.. Shandong electric power college journals. 13(5),8-10,(2010).

5. Xi Li, Lin Tang.. The software of time and space.22(3),212-214,(2006).

6. Moran Wang.. BeiJing: Electronic industry press. 2003.

7. Wang Yu,Fu Li, Shikun Zhang.. The computer technology and development. (2), 26-28,(2004).

8. Yinan Geng, Rong Zeng, Yu Li.. High voltage engineering. 36(6), 1501-1505, (2010).

9. Jiahong Chen, Jun Lv, Zhiyin Qian.. High voltage engineering. 35(12), 2891-2901, (2009).

10. Zhao Chun, Lei Mengfei, Su Jie. Electrotechnical Application. 33(21), 85-88(2014)

11. Dong Chen, Jianhua Liu, Chenxi Jia. Insulators and surge arresters. (5) ,8-12,(2011).

12. Haiyan Yuan, Zhengcai Fu, Bengang Wei.. Proseedings of the CSEE. (5): 111-117,(2009).

13. Yu Wang, Xishan Wen, Lei Lan.. High voltage engineering. 37(10), 2471-2476 (2011).

14. Richeng Luo, Wen Li, Zhiqian Li.. High voltage engineering. 41(8), 2780-2787 (2015). 